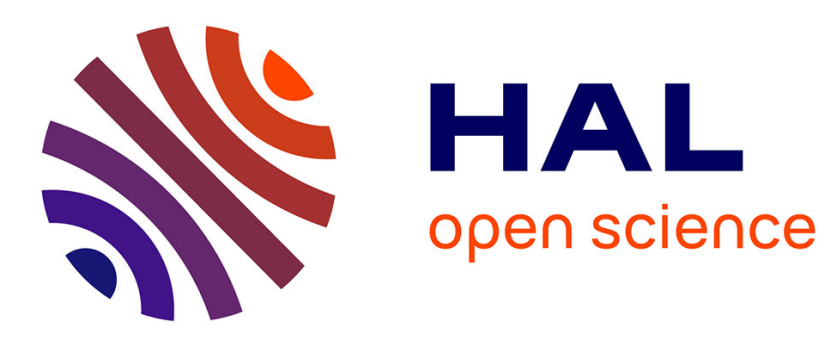

\title{
A minimal surface with one limit end and unbounded curvature
}

Martin Traizet

\section{To cite this version:}

Martin Traizet. A minimal surface with one limit end and unbounded curvature. Indiana University Mathematics Journal, 2012, 61 (3), pp.1325-1350. hal-00487921

\section{HAL Id: hal-00487921 \\ https://hal.science/hal-00487921}

Submitted on 31 May 2010

HAL is a multi-disciplinary open access archive for the deposit and dissemination of scientific research documents, whether they are published or not. The documents may come from teaching and research institutions in France or abroad, or from public or private research centers.
L'archive ouverte pluridisciplinaire HAL, est destinée au dépôt et à la diffusion de documents scientifiques de niveau recherche, publiés ou non, émanant des établissements d'enseignement et de recherche français ou étrangers, des laboratoires publics ou privés. 


\title{
A minimal surface with unbounded curvature
}

\author{
Martin Traizet
}

May 31, 2010

\section{Introduction}

The main goal of this paper is to construct a complete, embedded minimal surface in euclidean space $\mathbb{R}^{3}$ which has unbounded Gaussian curvature. Besides being a mathematical curiosity, this example is also of theoretical interests, as it illustrates some recent developments in the theory of properly embedded minimal surfaces of infinite topology.

Let me first explain why all examples of complete embedded minimal surfaces known so far have bounded Gaussian curvature. Almost all known examples fall into one of the following (non-disjoint) categories :

1. Finite total curvature minimal surfaces. Such a surface has a finite number of catenoidal or planar ends, so it must have bounded curvature.

2. Periodic minimal surfaces which have finite total curvature in the quotient. In this case, we know that the quotient is either compact (in the triplyperiodic case) or has a finite number of ends which are asymptotically flat, so again the curvature is bounded. This category contains the vast majority of known examples.

3. Properly embedded minimal surfaces with finite genus. By a recent result of Meeks, Perez and Ros [9], such a surface has bounded curvature. As examples which do not fit in one of the previous categories, we have the genus one helicoid [5] which has one end, and the Riemann examples with handles constructed in [4] which have infinitely many planar ends.

As far as I know, the only known examples which do not fit into one of these categories are the Saddle Towers with infinitely many ends [7] and the quasiperiodic examples constructed in [8]. Both are proven to have bounded Gaussian curvature (which actually requires some work).

In this paper we prove

Theorem 1 There exists a complete, properly embedded minimal surface in euclidean space $\mathbb{R}^{3}$ which has unbounded Gauss curvature. It has infinite genus, infinitely many catenoid type ends, and one limit-end. 
From the theoretical point of view, the most interesting feature of this example is its last property.

Collin, Kusner, Meeks and Rosenberg [1] have proven that a properly embedded minimal surface with infinitely many ends has at most two limit ends. Meeks, Perez and Ros [9] have proven that in the finite genus case, such a surface cannot have one limit end. The Riemann examples have genus zero and two limit ends. However, no example with just one limit end was known, so it seems interesting to construct an example to illustrate the theory. Of course, it must have infinite genus.

Let me point out that the existence of such an example is not completely unexpected. Indeed, at least heuristically, one can imagine how to construct one by inductively desingularizing a family of suitable catenoids. However, we don't have a general enough desingularization theorem at our disposal yet, and there are fantastic technicalities in trying to carry out such a construction. So the purpose of this paper is to contruct an example using another idea, in a somewhat more economical way.

Another remark is that if we relax the embeddedness condition, then there are plenty of known complete, immersed minimal surfaces with unbounded Gaussian curvature. For example, the example of Nadirashvili 10] of a complete minimal immersion in a ball certainly has unbounded curvature. Embeddedness is a strong constraint on the geometry of minimal surfaces.

Heuristically, our example is constructed inductively as follows. Start with the catenoid and stack a plane on top of it. Glue a finite number of catenoidal necks in between. After this first step one gets a Costa Hoffman Meeks surface with three ends. Then iterate this process infinitely many times, increasing the number of ends by one at each step. What we need to carry on this construction is a theorem which, from a minimal surface with $n$ ends, produces a minimal surface with one more end. This theorem is the main result of this paper and is stated in the next section.

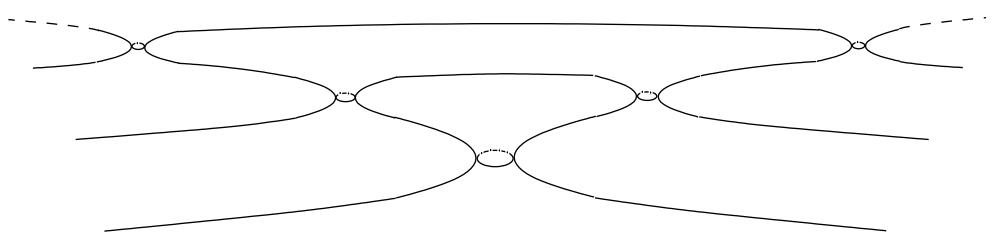

Figure 1: A sketch of the surface we get after two steps. Only two catenoidal necks have been represented at each level for clarity. 


\section{Main result}

Given an embedded minimal surface $M$ of finite total curvature in $\mathbb{R}^{3}$ with $n$ catenoidal ends, we denote $c_{1}(M), c_{2}(M), \cdots, c_{n}(M)$ the logarithmic growths of its ends, ordered from bottom to top.

Theorem 2 Let $M$ be an embedded minimal surface in $\mathbb{R}^{3}$ with finite total curvature, genus $G$ and $n$ horizontal catenoidal ends with logarithmic growths satisfying

$$
c_{1}(M)<c_{2}(M) \cdots<c_{n-1}(M)<0<c_{n}(M) .
$$

Assume that $M$ has a vertical plane of symmetry and non-degenerate Weierstrass Representation.

Consider an integer $m \geq 2$ such that

$$
m-1>\frac{c_{n}(M)}{\left|c_{n-1}(M)\right|} .
$$

There exists a one parameter family of embedded minimal surfaces $\left(M_{t}\right)_{0<t<\varepsilon}$ which has the following properties:

1. $M_{t}$ has finite total curvature, genus $G+m-1$ and $n+1$ catenoidal ends, whose logarithmic growths satisfy

$$
c_{1}\left(M_{t}\right)<c_{2}\left(M_{t}\right) \cdots<c_{n}\left(M_{t}\right)<0<c_{n+1}\left(M_{t}\right) .
$$

2. $M_{t}$ converges smoothly to $M$ on compact subsets of $\mathbb{R}^{3}$ when $t \rightarrow 0$.

3. $M_{t}$ has a vertical plane of symmetry and has non-degenerate Weierstrass Representation.

4. The maximum of the absolute value of the Gaussian curvature on $M_{t}$ is greater than $\frac{(m-1)^{2}}{2 c_{n}(M)^{2}}$.

This theorem will be proven in section 5 . The definition of "non-degenerate Weierstrass Representation" will be given in section 1 . Roughly speaking, this means that the space of deformations of $M$, keeping the vertical plane of symmetry, has the expected dimension, namely $n-1$. In particular, the catenoid has non-degenerate Weierstrass Representation.

Heuristically, $M_{t}$ is constructed by stacking a horizontal plane on top of $M$ and gluing $m$ catenoidal necks placed on a circle in between. When $t \rightarrow 0$, the catenoids drift off to infinity, which is why $M_{t}$ converges to $M$ on compact subsets of $\mathbb{R}^{3}$.

The catenoidal necks must all have waist radius equal to $r=\frac{c_{n}(M)}{m-1}$, they cannot be chosen freely. The Gaussian curvature of such a catenoid along its waist circle is equal to $-1 / r^{2}$, so this explains the last item of the theorem (the $1 / 2$ factor is here only because these catenoids only approximate the surface). 
By a simple evaluation of the vertical flux, we obtain, from the size of the catenoidal necks, the following values for the logarithmic growths of $M_{t}$

$$
\lim _{t \rightarrow 0} c_{k}\left(M_{t}\right)=\left\{\begin{array}{l}
c_{k}(M) \text { if } 1 \leq k \leq n-1 \\
\frac{-1}{m-1} c_{n}(M) \text { if } k=n \\
\frac{m}{m-1} c_{n}(M) \text { if } k=n+1
\end{array}\right.
$$

From this we see that the condition (11) ensures that $c_{n-1}\left(M_{t}\right)<c_{n}\left(M_{t}\right)$ as required. The construction is of course possible without this condition but will not produce an embedded minimal surface.

\section{Proof of theorem 1}

We construct inductively a sequence of minimal surfaces $\left(S_{n}\right)_{n \geq 2}$, an increasing sequence of balls $\left(B_{n}\right)_{n \geq 2}$ and a sequence of positive numbers $\left(C_{n}\right)_{n \geq 2}$ with the following properties:

1. Each $S_{n}$ is an embedded minimal surface of finite total curvature with $n$ catenoidal ends satisfying

$$
c_{1}\left(S_{n}\right)<c_{2}\left(S_{n}\right)<\cdots<c_{n-1}\left(S_{n}\right)<0<c_{n}\left(S_{n}\right)
$$

and with a vertical plane of symmetry and non-degenerate Weierstrass Representation.

2. For all $\ell \geq k \geq 2$, one has

$$
k-2<\sup _{S_{\ell} \cap B_{k}}|K|<C_{k} \quad \text { and } \quad \operatorname{Area}\left(S_{\ell} \cap B_{k}\right)<C_{k}
$$

where $K$ denotes the Gaussian curvature.

The process is initiated with $S_{2}$ equal to the standard catenoid, $B_{2}=B(0,2)$ and $C_{2}$ a suitable constant. Take $n \geq 2$ and assume that $S_{\ell}, B_{\ell}$ and $C_{\ell}$ have been constructed for all $\ell \leq n$, so that (3) is satisfied for all $2 \leq k \leq \ell \leq n$. We apply theorem 2 with $M=S_{n}$ and $m=m_{n}$ chosen large enough so that condition (11) is satisfied and $\frac{(m-1)^{2}}{2 c_{n}\left(S_{n}\right)^{2}}>n-1$. The output of the theorem is a family of minimal surfaces $\left(M_{t}\right)_{0<t<\varepsilon}$ which converges to $S_{n}$ on each $B_{k}$ for $k \leq n$. Hence we can choose $t$ small enough so that $S_{n+1}=M_{t}$ satisfies (3) for all $k \leq n$. By the last item of theorem 2, there are points on $S_{n+1}$ where $|K|>n-1$. We take a ball $B_{n+1}$ large enough to contain one such point, and containing $B_{n}$. Then we can choose a constant $C_{n+1}$ so that $S_{n+1}$ satisfies (3) for $k=n+1$ and we are done.

For each $k \geq 2$, the sequence $\left(S_{n} \cap B_{k}\right)_{n \geq k}$ has uniform curvature and area estimate, so has a subsequence which converges smoothly by standard compactness results (theorem 4.2.1 in [12]). By a diagonal process, the sequence $\left(S_{n}\right)_{n>2}$ has a subsequence which converges smoothly on each $B_{k}$, to a complete embedded minimal surface $S_{\infty}$. Now for all $k \geq 2, \sup _{S_{\infty} \cap B_{k}}|K| \geq k-2$, so $S_{\infty}$ has unbounded Gaussian curvature and the theorem is proven. 
Remark 1 All the catenoidal ends of $S_{\infty}$ have negative logarithmic growth.

In the above argument, we have chosen the sequence $\left(m_{n}\right)_{n \geq 2}$ so that the limit surface $S_{\infty}$ has unbounded Gaussian curvature, but can we choose it so that $S_{\infty}$ has bounded Gaussian curvature?

The sequence $\left(m_{n}\right)_{n \geq 2}$ must be chosen so that condition (1) is satisfied at each step. Using formula (2), we have

$$
\begin{aligned}
c_{n}\left(S_{n+1}\right) & \simeq \frac{-1}{m_{n}-1} c_{n}\left(S_{n}\right) \\
c_{n+1}\left(S_{n+1}\right) & \simeq \frac{m_{n}}{m_{n}-1} c_{n}\left(S_{n}\right)
\end{aligned}
$$

where $\simeq$ means that it can be chosen arbitrarily close by taking $t$ small enough. So condition (1) reads as $m_{n+1}-1>m_{n}$. Take an arbitrary sequence $\left(m_{n}\right)_{n \geq 2}$ satisfying

$$
m_{2} \geq 3 \quad \text { and } \quad \forall n \geq 2, \quad m_{n+1} \geq m_{n}+2
$$

By the above process, we obtain a sequence of minimal surface $\left(S_{n}\right)_{n \geq 2}$ which converges to an embedded minimal surface $S_{\infty}$ with infinitely many catenoidal ends. By induction, we have $m_{n} \geq 2 n-1$ and

$$
c_{n}\left(S_{n}\right) \simeq \prod_{i=2}^{n-1} \frac{m_{i}}{m_{i}-1} \leq \prod_{i=2}^{n-1}\left(1+\frac{1}{2 i-2}\right)=O(\sqrt{n}) .
$$

Hence, $\lim \frac{c_{n}\left(S_{n}\right)}{m_{n}-1}=0$. By the last item of theorem 2 this means that whatever the choice of the sequence $\left(m_{n}\right)_{n \geq 2}$ satisying (4), the minimal surface $S_{\infty}$ will have unbounded Gaussian curvature.

Also, we have

$$
\forall n \geq 2, \quad c_{n}\left(S_{\infty}\right) \simeq \frac{-1}{m_{n}-1} \prod_{i=2}^{n-1} \frac{m_{i}}{m_{i}-1}
$$

so depending on the choice of the sequence $\left(m_{n}\right)_{n \geq 2}$, the series $\sum c_{n}\left(S_{\infty}\right)$ can be convergent or divergent.

\section{Non-degenerate Weierstrass Representation}

Let $M$ be an embedded minimal surface in $\mathbb{R}^{3}$ with genus $G$ and $n$ horizontal catenoidal ends. Let $\left(\Sigma, g, \phi_{3}\right)$ be its Weierstrass Representation. Here $\Sigma$ is a compact Riemann surface, the Gauss map $g: \Sigma \rightarrow \overline{\mathbb{C}}=\mathbb{C} \cup\{\infty\}$ is a meromorphic function and the height differential $\phi_{3}$ is a meromorphic 1-form on $\Sigma$ with $n$ simple poles which we call $q_{1}, \cdots, q_{n}$. These points correspond to the ends of $M$ and are called the punctures. The degree of the Gauss map is $d=G+n-1$. Define

$$
\phi_{1}=\frac{1}{2}\left(g^{-1}-g\right) \phi_{3}, \quad \phi_{2}=\frac{i}{2}\left(g^{-1}+g\right) \phi_{3} .
$$


Our minimal surface $M$ is parametrized on $\Sigma \backslash\left\{q_{1}, \cdots, q_{n}\right\}$ by

$$
z \mapsto \operatorname{Re} \int_{z_{0}}^{z}\left(\phi_{1}, \phi_{2}, \phi_{3}\right) .
$$

We assume that $M$ has a vertical plane of symmetry. Without loss of generality we assume that $M$ is symmetric with respect to the plane $x_{2}=0$. On $\Sigma$, this symmetry corresponds to a antiholomorphic involution $\sigma$ such that $g \circ \sigma=\bar{g}$ and $\sigma^{*} \phi_{3}=\overline{\phi_{3}}$. Moreover, $\sigma$ fixes the punctures $q_{1}, \cdots, q_{n}$.

Definition 1 We say that the triple $\left(\Sigma, g, \phi_{3}\right)$ is $\sigma$-symmetric if there exists a antiholomorphic involution $\sigma: \Sigma \rightarrow \Sigma$ satisfying $g \circ \sigma=\bar{g}$ and $\sigma^{*} \phi_{3}=\overline{\phi_{3}}$.

Let us pretend we would like to deform $M$, keeping the vertical plane of symmetry. Let us write $\left(\Sigma_{0}, g_{0}, \phi_{3,0}\right)$ for the Weierstrass data of the minimal surface we are given. In the following sections, we count how many parameters are available for $\sigma$-symmetric deformation of the Weierstrass data and how many equations need to be solved. "Non-degenerate Weierstrass Representation" simply means that the jacobian matrix of equations with respect to parameters has maximal rank.

\subsection{Deformations of the Weierstrass data}

Let $\Sigma_{0}$ be a compact Riemann surface of genus $G$ and $g_{0}: \Sigma_{0} \rightarrow \overline{\mathbb{C}}$ be a meromorphic function of degree $d$. We see it as a branched covering of the Riemann sphere, and we would like to parametrize all deformations of $g_{0}$ by branched coverings $g: \Sigma \rightarrow \overline{\mathbb{C}}$. Two branched coverings $g: \Sigma \rightarrow \overline{\mathbb{C}}$ and $g^{\prime}: \Sigma^{\prime} \rightarrow \overline{\mathbb{C}}$ are said to be isomorphic if there exists a biholomorphic map $\psi: \Sigma \rightarrow \Sigma^{\prime}$ such that $g=g^{\prime} \circ \psi$.

In certain cases, the space of isomorphism classes of branched coverings are known to be smooth complex manifolds. For instance, a degree $d$ branched covering is said to be simple if each fiber contains at least $d-1$ points (so each fiber contains at most one branch point and it has branching order 1). The moduli space of simple branched coverings of given degree is called a Hurwitz space. It is an open complex manifold of dimension $2 G+2 d-2$. The list of branching values of $g$ provide local coordinates on this space. More generally, the moduli space of coverings of degree $d$ and with $n$ branch points form a smooth complex manifold of dimension $n$ [3].

Now if our covering has a branch point of branching order $k \geq 2$, when deforming it, this branch point may split into several smaller order branch points, whose branching orders sum up to $k$. It is not true anymore that the list of branching values provide local coordinates (see the example at the beginning of appendix B). The moduli space of branched covering of given degree, with no restriction on the branch points, is not a smooth complex manifold. It is singular at those coverings who have a branch point which is fixed by a nontrivial automorphism of the covering.

In the case of minimal surfaces, the punctures $q_{1}, \cdots, q_{n}$ are $n$ distinguished points on $\Sigma$ at which $g$ takes the value alternately 0 and $\infty$ with multiplicity one. 
We call the data $\left(\Sigma, g, q_{1}, \cdots, q_{n}\right)$ a marked covering. We say that two marked coverings $\left(\Sigma, g, q_{1}, \cdots, q_{n}\right)$ and $\left(\Sigma, g^{\prime}, q_{1}^{\prime}, \cdots, q_{n}^{\prime}\right)$ are isomorphic if there exists a biholomorphic map $\psi: \Sigma \rightarrow \Sigma^{\prime}$ such that $g=g^{\prime} \circ \psi$ and $\psi\left(x_{i}\right)=x_{i}^{\prime}$ for all $1 \leq i \leq n$.

The moduli space of marked coverings has a structure of complex manifold of dimension $2 G+2 d-2$. If, moreover, we require the coverings to be $\sigma$-symmetric, then it is a real manifold of dimension $2 G+2 d-2$. We present in appendix B one way to define local coordinates on this space.

As a conclusion, we can parametrize all $\sigma$-symmetric deformations of the marked covering $\left(\Sigma_{0}, g_{0}, q_{1}, \cdots, q_{n}\right)$ as $\left(\Sigma_{a}, g_{a}, q_{1}(a), \cdots, q_{n}(a)\right)$ with a parameter $a \in \mathbb{R}^{2 G+2 d-2}$ in a neighborhood of 0 . On each $\Sigma_{a}$ we have a antiholomorphic involution $\sigma$ such that $g_{a} \circ \sigma=\overline{g_{a}}$. The function $g$ takes alternately the value 0 and $\infty$ at $q_{1}(a), \ldots, q_{n}(a)$, with multiplicity one. These points are fixed by $\sigma$.

Then we would like to write all candidates for the height differential $\phi_{3}$ on $\Sigma_{a}$. It needs simple poles at the punctures $q_{1}, \cdots, q_{n}$. The most natural way to define such a meromorphic 1 -form is to prescribe residues and periods. Consider a canonical homology basis $A_{1}, \cdots, A_{G}, B_{1}, \cdots, B_{G}$ of $\Sigma_{a}$ (depending continuously on $a)$. Given complex parameters $c=\left(c_{1}, \cdots, c_{n-1}\right) \in \mathbb{C}^{n-1}$ and $\alpha=\left(\alpha_{1}, \cdots, \alpha_{G}\right) \in \mathbb{C}^{G}$ we define $\phi_{3}$ as the unique meromorphic 1-form on $\Sigma_{a}$ with simple poles at $q_{1}, \cdots, q_{n}$ and the following residues and $A$-periods :

$$
\begin{array}{cc}
\operatorname{Res}_{q_{i}} \phi_{3}=-c_{i} & 1 \leq i \leq n-1, \\
\int_{A_{i}} \phi_{3}=2 \pi i \alpha_{i} & 1 \leq i \leq G .
\end{array}
$$

The residue at $q_{n}$ is $c_{1}+\cdots+c_{n-1}$ by the Residue theorem. We would like $\phi_{3}$ to be $\sigma$-symmetric. This translates into simple conditions on the parameters $c$ and $\alpha$, provided we choose carefuly the homology basis.

Definition 2 Let $\Sigma$ be a Riemann surface with a antiholomorphic involution $\sigma$. We say that a canonical homology basis $\left\{A_{1}, \cdots, A_{G}, B_{1}, \cdots, B_{G}\right\}$ is $\sigma$ symmetric if there exists an action of $\sigma$ on $\{1, \cdots, G\}$ such that for all $1 \leq i \leq$ $G, \sigma\left(A_{i}\right) \sim-A_{\sigma(i)}$ and $\sigma\left(B_{i}\right) \sim B_{\sigma(i)}$.

One can construct a $\sigma$-symmetric canonical homology basis of $\Sigma=\Sigma_{a}$ as follows. The fixed set of $\sigma$ divides $\Sigma$ into two components $\Sigma^{+}$and $\Sigma^{-}$, so that $\sigma\left(\Sigma^{+}\right)=$ $\Sigma^{-}$. Let $G^{\prime}$ be the genus of $\Sigma^{+}$and $k$ the number of its boundary components. Then $G=2 G^{\prime}+k-1$. We consider $2 G^{\prime}$ cycles $A_{1}, \cdots, A_{G^{\prime}}, B_{1}, \cdots, B_{G^{\prime}}$ in $\Sigma^{+}$ such that the only non-zero intersection numbers are $A_{i} . B_{i}=1$. For $1 \leq i \leq G^{\prime}$, we define $A_{G^{\prime}+i}=-\sigma\left(A_{i}\right)$ and $B_{G^{\prime}+i}=\sigma\left(B_{i}\right)$. We take $B_{2 G^{\prime}+1}, \cdots, B_{2 G^{\prime}+k-1}$ to be the homology classes of all boundary components of $\Sigma^{+}$but one. For $1 \leq i \leq k-1$, we choose a cycle $A_{2 G^{\prime}+i}$ which intersects $B_{2 G^{\prime}+i}$ (with intersection number 1) and the remaining component of the boundary of $\Sigma^{+}$.

The condition $\sigma^{*} \phi_{3}=\overline{\phi_{3}}$ is equivalent to $\alpha_{\sigma(i)}=\overline{\alpha_{i}}$, for $1 \leq i \leq G$, and $c_{i} \in \mathbb{R}$ for $1 \leq i \leq n-1$. The parameters $c_{i}$ correspond geometrically to the logarithmic growths of the ends. The condition on $\alpha$ defines a space of real dimension $G$. 
Remark 2 Of course the Period Problem will require the parameters $\alpha_{i}$ to be real numbers, but it is better to leave this as an equation to be solved, else the dimension of the parameter space depends on $G^{\prime}$.

At this point we have defined a family of triples $\left(\Sigma_{a}, g_{a}, \phi_{3}\right)$ depending on the parameter $X=(a, c, \alpha)$. The parameter space has real dimension $5 G+3 n-5$. We write $X^{0}$ for the value of $X$ which gives the Weierstrass data $\left(\Sigma_{0}, g_{0}, \phi_{3,0}\right)$ of the minimal surface $M$ we were given. In general we will not write explicitely the dependance of objects on parameters, so we will write $\left(\Sigma, g, \phi_{3}\right)$, beeing understood that everything depends on the parameters.

\subsection{The equations}

In order to be define an immersed minimal surface, the triple $\left(\Sigma, g, \phi_{3}\right)$ must satisfy the following conditions :

1. At any zero of the height differential $\phi_{3}$, the Gauss map $g$ needs a zero or a pole, with the same multiplicity. We call this the zero/pole equation.

2. At each puncture $q_{1}, \cdots, q_{n}$, the residues of $\phi_{1}$ and $\phi_{2}$ must be real.

3. For all $\nu=1,2,3$ and $1 \leq i \leq G$ we need $\operatorname{Re} \int_{A_{i}} \phi_{\nu}=\operatorname{Re} \int_{B_{i}} \phi_{\nu}=0$.

Point 2 and 3 guarantee that (5) is well defined, and point 1 that it is an immersion. Let us count how many equations we have to solve, taking into account the $\sigma$-symmetry.

- Regarding point 2 , the residues of $\phi_{1}$ are real and the residues of $\phi_{2}$ are imaginary by symmetry. Also, the residue of $\phi_{2}$ at $q_{j}$ is $\frac{i}{2} \operatorname{Res} g^{-1} \phi_{3}$ if $g\left(q_{j}\right)=0$, and $\frac{i}{2} \operatorname{Res} g \phi_{3}$ if $g\left(q_{j}\right)=\infty$. Provided point 1 is satisfied, the only poles of $g \phi_{3}$ and $g^{-1} \phi_{3}$ are at the punctures. Applying the Residue Theorem to $g \phi_{3}$ and $g^{-1} \phi_{3}$, it suffices to solve the equation $\operatorname{Res}_{q_{j}} \phi_{2}=0$ for $1 \leq i \leq n-2$. Therefore, point 2 counts as $n-2$ real equations.

- Regarding the Period Problem, we have the symmetries $\sigma^{*} \phi_{\nu}=(-1)^{\nu+1} \overline{\phi_{\nu}}$, $\nu=1,2,3$. From this we obtain, for $1 \leq i \leq G$,

$$
\begin{gathered}
\operatorname{Re} \int_{A_{\sigma(i)}} \phi_{\nu}=(-1)^{\nu} \operatorname{Re} \int_{A_{i}} \phi_{\nu} \\
\operatorname{Re} \int_{B_{\sigma(i)}} \phi_{\nu}=(-1)^{\nu+1} \operatorname{Re} \int_{B_{i}} \phi_{\nu} .
\end{gathered}
$$

So the period problem reduces to $3 G$ real equations. Explicitely, for the canonical basis that we defined in the previous section, these equations are

$$
\operatorname{Re} \int_{A_{i}} \phi_{\nu}=0, \quad 1 \leq i \leq G^{\prime}, \quad \nu=1,3,
$$




$$
\begin{gathered}
\operatorname{Re} \int_{B_{i}} \phi_{\nu}=0, \quad G^{\prime}+1 \leq i \leq G, \quad \nu=1,3, \\
\operatorname{Re} \int_{A_{i}} \phi_{2}=0, \quad G^{\prime}+1 \leq i \leq G \\
\operatorname{Re} \int_{B_{i}} \phi_{2}=0, \quad 1 \leq i \leq G^{\prime} .
\end{gathered}
$$

- Regarding point 1 , the height differential $\phi_{3}$ has $2 G+n-2$ zeros, counting multiplicity. Let us first assume that the zeros of $\phi_{3,0}$ are simple. Then this remains true when the parameter $X$ is close to $X^{0}$, and we may label them $\zeta_{1}(X), \cdots, \zeta_{2 G+n-2}(X)$ so that they depend continuously on $X$. If $g_{0}$ has a zero (resp. a pole) at $\zeta_{i}\left(X^{0}\right)$, we need to solve the equation $g_{a}\left(\zeta_{i}(X)\right)=0$ (resp. $g(a)^{-1}\left(\zeta_{i}(X)\right)=0$ ). Taking into account the $\sigma$-symmetry (which implies that the set of zeros of $\phi_{3}$ is invariant by $\sigma$ ), these are $2 G+n-2$ real equations (the equations are either real or conjugate by pairs).

- In case $\phi_{3,0}$ has a zero of multiplicity $k \geq 2$ at some point $\zeta$, the problem is that this zero may split into several zeros of smaller multiplicity when we deform the Weierstrass data. Let us assume for example that $g_{0}$ has a zero (of multiplicity $k$ ) at $\zeta$. By the very definition of $\left(\Sigma_{a}, g_{a}\right)$, there is a local coordinate $z$ on $\Sigma_{a}$ such that

$$
g_{a}(z)=z^{k}+\sum_{i=0}^{k-1} a_{i} z^{i}
$$

where the coefficients $a_{i}$ are components of the parameter vector $a$ (and actually $\left.a_{k-1}=0\right)$. By the Weierstrass Preparation Theorem, we can write

$$
\phi_{3}=f(X, z)\left(z^{k}+\sum_{i=0}^{k-1} b_{i}(X) z^{i}\right) d z
$$

where the coefficients $b_{i}(X)$ are holomorphic functions of $X$. The polynomial $z^{k}+\sum b_{i} z^{i}$ is called the Weierstrass Polynomial of $\phi_{3}$, it depends on the choice of the coordinate $z$. We solve the equations $b_{i}(X)=a_{i}$, $0 \leq i \leq k-1$. Taking into account the $\sigma$-symmetry we have the same number of equations as before.

Everything together, we have to solve $5 G+2 n-4$ real equations. Let us write these equations as $\mathcal{F}(X)=0$. Recall that the dimension of the parameter space is $5 G+3 n-5$.

Definition 3 We say that $M$ has non-degenerate Weierstrass Representation if the differential of $\mathcal{F}$ at $X^{0}$ is onto. Equivalently, its kernel has dimension $n-1$. 
Example : the catenoid has non-degenerate Weierstrass Representation, because in this case, $G=0$ and $n=2$, so the parameter space has dimension 1 and there are no equations to solve. As expected, the catenoid comes into a one-parameter family (by scaling).

As a straightforward application of the implicit function theorem, we have

Proposition 1 If $M$ has non-degenerate Weierstrass Representation, then it can be deformed into a family of non-congruent minimal surfaces depending on $n-1$ parameters, all symmetric with respect to the plane $x_{2}=0$.

Remark 3 We do not require that the parameters in this deformation are the logarithmic growths of the ends, so this notion of non-degeneracy should be weaker than the usual non-degeneracy defined in term of Jacobi fields [11, altough I have not been able to prove that one of them implies the other.

\section{$5 \quad$ Proof of theorem 2}

We construct the family of minimal surfaces $\left(M_{t}\right)_{0<t<\varepsilon}$ by writing down candidates for its Weierstrass Representation and then solving the zero/pole equation and the Period Problem. We define the Riemann surface and the Gauss map by opening nodes. The height differential $\phi_{3}$ is defined by prescribing periods and residues. The equations are solved using the implicit function theorem, using the fact that $M$ has non-degenerate Weierstrass Representation.

\subsection{Opening nodes}

Since $M$ has non-degenerate Weierstrass Representation, we are given in particular a family of branched coverings $(\Sigma, g)=\left(\Sigma_{a}, g_{a}\right)$ depending on the parameter a. Let $q_{n}=q_{n}(a) \in \Sigma_{a}$ be the point which corresponds to the top end. Without loss of generality we may assume that $g\left(q_{n}\right)=0$.

We consider two copies of the complex plane, denoted $\mathbb{C}^{-}$and $\mathbb{C}^{+}$. We choose $m$ distinct, non-zero points $p_{1}^{-}, \cdots, p_{m}^{-}$in $\mathbb{C}^{-}$and $m$ distinct points $p_{1}^{+}, \cdots, p_{m}^{+}$in $\mathbb{C}^{+}$. Identify the point $q_{n}$ with the point 0 in $\mathbb{C}^{-}$. For $1 \leq i \leq m$, identify the point $p_{i}^{-}$with the point $p_{i}^{+}$. This defines a Riemann surfaces with $m+1$ nodes which we call $\widetilde{\Sigma}_{0}$.

We define two meromorphic functions $g^{-}$on $\mathbb{C}^{-}$and $g^{+}$on $\mathbb{C}^{+}$by

$$
\begin{gathered}
g^{-}(z)=\frac{\beta_{0}^{-}}{z}+\sum_{i=1}^{m} \frac{\beta_{i}^{-}}{z-p_{i}^{-}} \\
g^{+}(z)=\sum_{i=1}^{m} \frac{\beta_{i}^{+}}{z-p_{i}^{+}} .
\end{gathered}
$$

Here $\beta_{0}^{-}, \cdots, \beta_{m}^{-}$and $\beta_{1}^{+}, \cdots, \beta_{m}^{+}$are non-zero complex parameters. We write $\beta^{-}=\left(\beta_{0}^{-}, \cdots, \beta_{m}^{-}\right), \beta^{+}=\left(\beta_{1}^{+}, \cdots, \beta_{m}^{+}\right)$and $p^{ \pm}=\left(p_{1}^{ \pm}, \cdots, p_{m}^{ \pm}\right)$. 
Since $g$ has a simple zero at $q_{n}$ and $g^{ \pm}$have simple poles, we can fix a small number $0<\epsilon<1$ such that $v_{0}^{-}:=g$ is a diffeomorphism from a small neighborhood $U_{0}^{-}$of $q_{n}$ to the disk $D(0, \epsilon), v_{0}^{+}:=1 / g^{-}$is a diffeomorphism from a small neighborhood $U_{0}^{+}$of 0 in $\mathbb{C}^{-}$to $D(0, \epsilon)$ and for each $i=1, \cdots, m$, $v_{i}^{ \pm}:=1 / g^{ \pm}$is a diffeomorphism from a small neighborhood $U_{i}^{ \pm}$of $p_{i}^{ \pm}$in $\mathbb{C}^{ \pm}$to $D(0, \epsilon)$. We use $v_{i}^{ \pm}$as local complex coordinates to open nodes.

Consider a real parameter $t$ such that $0<t<\epsilon^{2}$. We remove the disk $\left|v_{0}^{-}\right|<\frac{t}{\epsilon}$ from $U_{0}^{-}$and the disk $\left|v_{0}^{+}\right|<\frac{t}{\epsilon}$ from $U_{0}^{+}$. We identify the point $z \in U_{0}^{-}$with the point $z^{\prime} \in U_{0}^{+}$such that $v_{0}^{-}(z) v_{0}^{+}\left(z^{\prime}\right)=t$. This is equivalent to

$$
g(z)=t g^{-}\left(z^{\prime}\right)
$$

For each $1 \leq i \leq m$, we remove the disks $\left|v_{i}^{-}\right|<\frac{t^{2}}{\epsilon}$ from $U_{i}^{-}$and $\left|v_{i}^{+}\right|<\frac{t^{2}}{\epsilon}$ from $U_{i}^{+}$. We identify the point $z \in U_{i}^{-}$with the point $z^{\prime} \in U_{i}^{+}$such that $v_{i}^{-}(z) v_{i}^{+}(z)=t^{2}$. This is equivalent to

$$
t g^{-}(z)=\frac{1}{t g^{+}(z)}
$$

This defines a Riemann surface. We compactify it by adding the points at infinity in $\mathbb{C}^{-}$and $\mathbb{C}^{+}$and call it $\widetilde{\Sigma}$. Its genus is $\widetilde{G}=G+m-1$.

By a slight abuse of language, we will denote by $\Sigma \subset \widetilde{\Sigma}, \mathbb{C}^{-} \subset \widetilde{\Sigma}$ and $\mathbb{C}^{+} \subset \widetilde{\Sigma}$ the domains $\Sigma, \mathbb{C}^{-}$and $\mathbb{C}^{+}$minus the disks that were removed when opening nodes.

We define the Gauss map $\widetilde{g}$ on $\widetilde{\Sigma}$ by

$$
\widetilde{g}(z)= \begin{cases}g(z) & \text { if } z \in \Sigma \\ t g^{-}(z) & \text { if } z \in \mathbb{C}^{-} \\ \frac{1}{\operatorname{tg}(z)} & \text { if } z \in \mathbb{C}^{+}\end{cases}
$$

This is a well defined meromorphic function on $\widetilde{\Sigma}$ because $\widetilde{g}(z)=\widetilde{g}\left(z^{\prime}\right)$ whenever $z$ and $z^{\prime}$ are identified.

Next we would like $(\widetilde{\Sigma}, \widetilde{g})$ to have the required symmetry. By hypothesis, $\Sigma$ does have a antiholomorphic involution $\sigma$ such that $g \circ \sigma=\bar{g}$. Let $V$ be the linear subspace of $\mathbb{C}^{m-1}$ defined by $z_{m-i}=\overline{z_{i}}$ for $1 \leq i \leq m-1$. This is a real vector space of dimension $m-1$. We require that $\beta^{-} \in \mathbb{R} \times V \times \mathbb{R}$, and $\beta^{+}, p^{+}$ and $p^{-}$all belong to $V \times \mathbb{R}$. Under these assumptions, we have $g^{-}(\bar{z})=\overline{g^{-}(z)}$ and $g^{+}(\bar{z})=\overline{g^{+}(z)}$. We define $\widetilde{\sigma}: \widetilde{\Sigma} \rightarrow \widetilde{\Sigma}$ by $\widetilde{\sigma}=\sigma$ on $\Sigma$ and $\widetilde{\sigma}(z)=\bar{z}$ on $\mathbb{C}^{-}$and $\mathbb{C}^{+}$. Then thanks to the fact that $t$ is real, $\widetilde{\sigma}$ is well defined on $\widetilde{\Sigma}$, and $\widetilde{g} \circ \widetilde{\sigma}=\overline{\widetilde{g}}$. We will write $\sigma$ for $\widetilde{\sigma}$ as no confusion can occur.

\subsection{The height differential}

As in section 4.1, we define the height differential on $\widetilde{\Sigma}$ by prescribing periods and residues, so we need to define a canonical homology basis of $\widetilde{\Sigma}$. The cycles $A_{1}, \cdots, A_{G}, B_{1}, \cdots, B_{G}$ on $\Sigma$ define us $2 G$ cycles on $\widetilde{\Sigma}$. For $1 \leq i \leq m-1$, let 
$A_{G+i}$ be the homology class of the circle $C\left(p_{i}^{+}, \epsilon\right)$ with the positive orientation. This circle is homologous to the circle $C\left(p_{i}^{-}, \epsilon\right)$ with the negative orientation.

For $1 \leq i \leq \frac{m}{2}$, we define $B_{G+i}$ as the composition of the following paths :

1. a path from the point $v_{m}^{+}=-\epsilon$ to the point $v_{i}^{+}=\epsilon$ in $\mathbb{C}^{+}$,

2. the segment from $v_{i}^{+}=\epsilon$ to $v_{i}^{+}=\frac{t^{2}}{\epsilon}$,

3. a path from the point $v_{i}^{-}=\epsilon$ to the point $v_{m}^{-}=-\epsilon$ in $\mathbb{C}^{-}$,

4. the segment from $v_{m}^{-}=-\epsilon$ to $v_{m}^{-}=-\frac{t^{2}}{\epsilon}$.

In point 1 and 3 , the curves must depend continuously on parameters, and must avoid all disks around the nodes. In particular, if $i=\frac{m}{2}$, we cannot take the straight segment in point 3 because it goes through the origin (which has been removed from $\mathbb{C}^{-}$). Also, this definition only makes sense for $t \neq 0$. For $\frac{m}{2}<i \leq m-1$, we define $B_{G+i}$ as $\sigma\left(B_{G+m-i}\right)$. Then $A_{1}, \cdots, A_{\widetilde{G}}, B_{1}, \cdots, B_{\widetilde{G}}$ is a $\sigma$-symmetric canonical homology basis of $\widetilde{\Sigma}$.

Let $\infty^{-}$and $\infty^{+}$denote the point at infinity in $\mathbb{C}^{-}$and $\mathbb{C}^{+}$. The punctures (corresponding to the $n+1$ catenoidal ends) are at $q_{1}, \cdots, q_{n-1}, \infty^{-}$and $\infty^{+}$. We define the height differential $\widetilde{\phi}_{3}$ on $\widetilde{\Sigma}$ as in section 4.1 by prescribing its $A$-periods and its residues at all punctures but one. Actually, by the residue theorem, prescribing the residue at $\infty^{+}$is the same as prescribing the period on the circle $C\left(p_{m}^{+}, \epsilon\right)$. So we define $\widetilde{\phi}_{3}$ on $\widetilde{\Sigma}$ as the unique meromorphic 1 -form with simple poles at the punctures with the following residues and periods :

$$
\begin{gathered}
\int_{A_{j}} \widetilde{\phi}_{3}=2 \pi i \alpha_{j}, \quad 1 \leq j \leq G \\
\operatorname{Res}_{q_{i}} \widetilde{\phi}_{3}=-c_{i}, \quad 1 \leq i \leq n-1, \\
\int_{C\left(p_{j}^{+}, \epsilon\right)} \widetilde{\phi}_{3}=2 \pi i \gamma_{j}, \quad 1 \leq j \leq m
\end{gathered}
$$

The parameters $c=\left(c_{1}, \cdots, c_{n-1}\right)$ and $\alpha=\left(\alpha_{1}, \cdots, \alpha_{G}\right)$ are as in section 4.1. The parameter $\gamma=\left(\gamma_{1}, \cdots, \gamma_{m}\right)$ is in the vector space $V \times \mathbb{R}$ defined above, so that $\widetilde{\phi}_{3}$ satisfies $\sigma^{*} \widetilde{\phi}_{3}={\widetilde{\phi_{3}}}_{3}$. By the residue theorem in $\mathbb{C}^{+}$and $\widetilde{\Sigma}$, we have

$$
\begin{gathered}
\operatorname{Res}_{\infty^{+}} \widetilde{\phi}_{3}=-\sum_{i=1}^{m} \gamma_{j}, \\
\operatorname{Res}_{\infty^{-}} \widetilde{\phi}_{3}=\sum_{j=1}^{n-1} c_{j}+\sum_{j=1}^{m} \gamma_{j} .
\end{gathered}
$$

By standard results [2, 6], on a Riemann surface with nodes, the notion of holomorphic (or meromorphic) 1-form must be replaced by that of a regular differential, which means that it has simple poles on each side of each node, 
with opposite residues. In the case at hand, this means that when $t=0$, $\widetilde{\phi}_{3}$ is meromorphic in $\Sigma, \mathbb{C}^{-}$and $\mathbb{C}^{+}$, with simples poles at $q_{1}, \cdots, q_{n}$ in $\Sigma$, simple poles at $0, p_{1}^{-}, \cdots, p_{m}^{-}$and $\infty^{-}$in $\mathbb{C}^{-}$and simples poles at $p_{1}^{+}, \cdots, p_{m}^{+}$ and $\infty^{+}$in $\mathbb{C}^{+}$. The above period and residue prescription define $\widetilde{\phi}_{3}$ uniquely, and moreover $\widetilde{\phi}_{3}$ depends analytically (away from the poles) on $t$ and all other parameters. Given the residues and periods of $\widetilde{\phi}_{3}$, we have, when $t=0$ :

$$
\widetilde{\phi}_{3}=\left\{\begin{array}{l}
\phi_{3} \quad \text { in } \Sigma, \\
-\sum_{i=1}^{n-1} c_{i} \frac{d z}{z}-\sum_{i=1}^{m} \frac{\gamma_{i}}{z-p_{i}^{-}} d z \quad \text { in } \mathbb{C}^{-}, \\
\sum_{i=1}^{m^{m}} \frac{\gamma_{i}}{z-p_{i}^{+}} d z \quad \text { in } \mathbb{C}^{+} .
\end{array}\right.
$$

The key point is that when $t=0$, the restriction of $\widetilde{g}$ and $\widetilde{\phi}_{3}$ to $\Sigma$ are $g$ and $\phi_{3}$ as defined in section 4.1. This allow us to use the non-degeneracy hypothesis.

\subsection{Central value of the parameters}

All the parameters that we have introduced vary in a neighborhood of a central value, which is the point at which we will apply the implicit function theorem.

The central value of the $t$ parameter is zero. The central value of the parameters $a, \alpha_{1}, \cdots, \alpha_{G}$ and $c_{1}, \cdots, c_{n-1}$ are the values such that $\left(\Sigma, g, \phi_{3}\right)=$ $\left(\Sigma_{0}, g_{0}, \phi_{3,0}\right)$ is the Weierstrass Representation of our given minimal surface $M$.

The central value of the other parameters will be found by solving the equations, but it may help the reader to give it here. Without loss of generality, we may assume by scaling that the logarithmic growth of the top end of $M$ is $c_{n}=1$. The central value of the parameters $\beta^{+}, \beta^{-}$and $\gamma$ are then given by

$$
\beta_{0}^{-}=-1, \quad \beta_{i}^{+}=\beta_{i}^{-}=\gamma_{i}=\frac{1}{m-1}, \quad 1 \leq i \leq m
$$

The central value of the parameters $p^{ \pm}$are given by

$$
p_{i}^{+}=\omega^{-i}, p_{i}^{-}=\omega^{i}, \quad 1 \leq i \leq m
$$

where $\omega=e^{2 \pi i / m}$ is a primitive $m$-th root of unity.

When all parameters have their central value, we have

$$
\begin{gathered}
g^{-}(z)=\frac{-1}{z}+\frac{1}{m-1} \sum_{i=1}^{m} \frac{1}{z-\omega^{i}}=\frac{-1}{z}+\frac{m z^{m-1}}{(m-1)\left(z^{m}-1\right)} \\
g^{+}(z)=\frac{1}{m-1} \sum_{i=1}^{m} \frac{1}{z-\omega^{i}}=\frac{m z^{m-1}}{(m-1)\left(z^{m}-1\right)} .
\end{gathered}
$$

Moreover, $\widetilde{\phi}_{3}=-g^{-}(z) d z$ in $\mathbb{C}^{-}$and $\widetilde{\phi}_{3}=g^{+}(z) d z$ in $\mathbb{C}^{+}$. 


\subsection{The equations}

As in section 4.2, we have to solve the following equations to ensure that the Weierstrass data $\left(\widetilde{\Sigma}, \widetilde{g}, \widetilde{\phi}_{3}\right)$ defines a minimal immersion.

1a. At each zero of $\widetilde{\phi}_{3}$ in $\Sigma \subset \widetilde{\Sigma}$, the gauss map $\widetilde{g}=g$ needs a zero or a pole, with the same multiplicity.

1b. At each zero of $\widetilde{\phi}_{3}$ in $\mathbb{C}^{ \pm}, g^{ \pm}$needs a zero with the same multiplicity.

2a. At each puncture $q_{1}, \cdots q_{n-2}$, the residues of $\phi_{1}$ and $\phi_{2}$ must be real.

2b. The residues of $\phi_{1}$ and $\phi_{2}$ at $\infty^{+}$must be real.

3a. $\operatorname{Re} \int_{A_{i}} \widetilde{\phi}_{\nu}=\operatorname{Re} \int_{B_{i}} \widetilde{\phi}_{\nu}=0 \quad$ for $1 \leq i \leq G, \nu=1,2,3$.

3b. $\operatorname{Re} \int_{A_{G+i}} \widetilde{\phi}_{\nu}=\operatorname{Re} \int_{B_{G+i}} \widetilde{\phi}_{\nu}=0 \quad$ for $1 \leq i \leq m-1, \nu=1,2,3$.

In the following points, we study how each of these equations extend to $t=0$.

- Let $\mathcal{F}$ be the collection of the equations in point $1 \mathrm{a}, 2 \mathrm{a}$ and $3 \mathrm{a}$. When $t=0$, the restriction of $\widetilde{g}$ and $\widetilde{\phi}_{3}$ to $\Sigma$ are $g$ and $\phi_{3}$, so these equations are exactly the same as the ones in section 4.2. So when $t=0$, the function $\mathcal{F}$ equals the function $\mathcal{F}$ defined in section 4.2. The non-degeneracy hypothesis will take care of this equation.

- Regarding point $1 \mathrm{~b}$, at the central value of the parameters, $\widetilde{\phi}_{3}$ has $m$ simple zeros in $\mathbb{C}^{-}$, so this remains true for nearby values of the parameters. We may call these zeros $\zeta_{1}, \cdots, \zeta_{m}$ so that $\sigma\left(\zeta_{i}\right)=\zeta_{m+1-i}$. Let $\mathcal{Z}^{-}=$ $\left(g^{-}\left(\zeta_{1}\right), \cdots, g^{-}\left(\zeta_{m}\right)\right)$ (the letter $\mathcal{Z}$ stands for "zero"). This is an analytic function of all parameters. Moreover, $\mathcal{Z}_{m+1-i}^{-}=\overline{\mathcal{Z}_{i}^{-}}$so $\mathcal{Z}^{-}$takes value in a real space of dimension $m$.

- At the central value, $\widetilde{\phi}_{3}$ has one zero of multiplicity $m-1$ at the origin. We may write $g^{+}(z)=\frac{P(z)}{Q(z)}$ where $P$ is a unitary polynomial of degree $m-1$ whose coefficients are (polynomial) functions of the parameters $\beta_{i}^{+}$ and $p_{i}^{+}$. Let $R$ be the Weierstrass polynomial of $\widetilde{\phi}_{3}$ in a neighborhood of 0 . We define $\mathcal{Z}^{+}=P-R$, this is a real polynomial of degree $m-2$ whose coefficients are analytic functions of all parameters. We need to solve the equation $\mathcal{Z}^{+}=0$.

- Regarding the $A$-periods in point $3 \mathrm{~b}$, we define

$$
\begin{gathered}
\mathcal{V}_{j}^{A}=\operatorname{Re} \int_{C\left(p_{j}^{+}, \epsilon\right)} \widetilde{\phi}_{3}=-2 \pi \operatorname{Im}\left(\gamma_{j}\right), \quad 1 \leq j \leq m-1, \\
\mathcal{H}_{j}^{A}=\frac{1}{t}\left(\operatorname{Re} \int_{C\left(p_{j}^{+}, \epsilon\right)} \widetilde{\phi}_{1}+i \operatorname{Re} \int_{C\left(p_{j}^{+}, \epsilon\right)} \widetilde{\phi}_{2}\right), \quad 1 \leq j \leq m .
\end{gathered}
$$


(The letters $\mathcal{V}$ stand for "vertical", $\mathcal{H}$ for "horizontal" and $A$ for " $A$ cycles".) Note that the equation $\mathcal{H}_{A}=0$ takes care of point $2 \mathrm{~b}$ by the Residue Theorem. The symmetry gives $\mathcal{H}_{m-i}^{A}=-\overline{\mathcal{H}_{i}^{A}}$, so $\mathcal{H}^{A}$ takes value in a space of real dimension $m$. The function $\mathcal{H}^{A}$ extends analytically to $t=0$ by the following computation :

$$
\begin{aligned}
\mathcal{H}_{i}^{A} & =\frac{1}{2 t}\left(\overline{\int_{C\left(p_{i}^{+}, \epsilon\right)} \widetilde{g}^{-1} \widetilde{\phi}_{3}}-\int_{C\left(p_{i}^{+}, \epsilon\right)} \widetilde{g} \widetilde{\phi}_{3}\right) \\
& =\frac{1}{2 t}\left(\overline{\int_{C\left(p_{i}^{+}, \epsilon\right)} \widetilde{g}^{-1} \widetilde{\phi}_{3}}+\int_{C\left(p_{i}^{-}, \epsilon\right)} \widetilde{g} \widetilde{\phi}_{3}\right) \\
& =\frac{1}{2}\left(\overline{\int_{C\left(p_{i}^{+}, \epsilon\right)} g^{+} \widetilde{\phi}_{3}}+\int_{C\left(p_{i}^{-}, \epsilon\right)} g^{\left.-\widetilde{\phi}_{3}\right)}\right.
\end{aligned}
$$

- By lemma 1 in [14], the function $\int_{B_{G+i}} \widetilde{\phi}_{3}-\left(\gamma_{i}-\gamma_{m}\right) \log t^{2}$ extends to an analytic function of all parameters at $t=0$. We make the change of variable $t=\exp \left(\frac{-1}{\tau^{2}}\right)$ where $\tau$ is a real parameter in a neighborhood of zero. We define the renormalised vertical $B$-periods as

$$
\mathcal{V}_{i}^{B}=\tau^{2} \operatorname{Re} \int_{B_{G+i}} \widetilde{\phi}_{3}, \quad 1 \leq i \leq m-1 .
$$

Then $\mathcal{V}^{B}=\left(\mathcal{V}_{1}^{B}, \cdots, \mathcal{V}_{m-1}^{B}\right)$ extends as a smooth function of all parameters at $\tau=0$, with value

$$
\mathcal{V}_{i}^{B}=-2 \operatorname{Re}\left(\gamma_{i}-\gamma_{m}\right) \quad \text { at } \tau=0 .
$$

The symmetry gives $\mathcal{V}_{m-i}^{B}=\mathcal{V}_{i}^{B}$.

- We define the renormalised horizontal $B$-periods as

$$
\mathcal{H}_{j}^{B}=t\left(\operatorname{Re} \int_{B_{G+j}} \widetilde{\phi}_{1}+i \operatorname{Re} \int_{B_{G+j}} \widetilde{\phi}_{2}\right), \quad 1 \leq j \leq m-1
$$

By lemma 2 in [14], $\mathcal{H}^{B}=\left(\mathcal{H}_{1}^{B}, \cdots, \mathcal{H}_{m-1}^{B}\right)$ extends to a smooth function of all parameters at $\tau=0$ with value

$$
\mathcal{H}_{i}^{B}=\frac{1}{2} \overline{\int_{p_{i}^{-}}^{p_{m}^{-}} \frac{\widetilde{\phi}_{3}}{g^{-}}}-\frac{1}{2} \int_{p_{m}^{+}}^{p_{i}^{+}} \frac{\widetilde{\phi}_{3}}{g^{+}} \text {at } \tau=0 .
$$

The symmetry gives $\mathcal{H}_{m-i}^{B}=\overline{\mathcal{H}_{i}^{B}}$ so $\mathcal{H}^{B}$ takes value in the space $V$.

\subsection{Solving the equations}

Let $X$ be the collection of all parameters but $\tau$. We denote by $X^{0}$ the central value of the parameters. Let $\widetilde{\mathcal{F}}(\tau, X)$ be the collection of the equations that we have to solve, namely $\widetilde{\mathcal{F}}=\left(\mathcal{F}, \mathcal{Z}^{-}, \mathcal{Z}^{+}, \mathcal{V}^{A}, \mathcal{H}^{A}, \mathcal{V}^{B}, \mathcal{H}^{B}\right)$. We want to solve the equation $\widetilde{\mathcal{F}}(\tau, X)$ to get $X$ as an implicit function of $\tau$. 
Lemma 1 We have $\widetilde{\mathcal{F}}\left(0, X^{0}\right)=0$, and the partial differential of $\widetilde{\mathcal{F}}$ with respect to $X$ at $\left(0, X^{0}\right)$ is onto.

Proof : we make a change of parameters so that the partial differential is triangular by blocks. Let

$$
\begin{gathered}
\gamma_{i}=\gamma_{m}+\dot{\gamma}_{i}, \quad 1 \leq i \leq m-1, \\
\beta_{i}^{-}=\gamma_{i}+\dot{\beta}_{i}^{-}, \quad 1 \leq i \leq m, \\
\beta_{0}^{-}=\sum_{i=1}^{n-1} c_{i}+\dot{\beta}_{0}^{-} \\
\beta_{i}^{+}=\gamma_{i}+\dot{\beta}_{i}^{+}, \quad 1 \leq i \leq m \\
p_{i}^{+}=\overline{p_{i}^{-}}+\dot{p}_{i}^{+}, \quad 1 \leq i \leq m .
\end{gathered}
$$

We write $\dot{\gamma}=\left(\dot{\gamma}_{1}, \cdots, \dot{\gamma}_{m-1}\right) \in V, \dot{\beta}^{-}=\left(\dot{\beta}_{0}^{-}, \cdots, \dot{\beta}_{m}^{-}\right) \in \mathbb{R} \times V \times \mathbb{R}, \dot{\beta}^{+}=$ $\left(\dot{\beta}_{1}^{+}, \cdots, \dot{\beta}_{m}^{+}\right) \in V \times \mathbb{R}$ and $\dot{p}^{+}=\left(\dot{p}_{1}^{+}, \cdots, \dot{p}_{m}^{+}\right) \in V \times \mathbb{R}$. The central value of each of these new parameters is 0 . Now the parameters are $a, \alpha, c, \gamma_{m}, \dot{\gamma}, \dot{\beta}^{-}$, $\dot{\beta}^{+}, p^{-}, \dot{p}^{+}$and $\tau$. In the following points, we evaluate the partial differential at the central value of each equation with respect to all these parameters except $\tau$. At the same time we check that the equations are satisfied at the central value of the parameters.

- By the non-degeneracy hypothesis, the partial differential of $\mathcal{F}$ with respect to the parameters $(a, \alpha, c)$ is onto. Moreover, its partial derivative with respect to all other parameters is zero.

- The partial differential of $\mathcal{Z}^{-}$with respect to $\dot{\beta}^{-}$is onto, and all other partial derivatives of $\mathcal{Z}^{-}$are zero.

Indeed, when $t=0$, the zeros of $\widetilde{\phi}_{3}$ do not depend on $\beta^{-}$anymore, so $\mathcal{Z}^{-}$ is a linear function of $\dot{\beta}^{-}$. If $\dot{\beta}^{-}$is in the kernel of the partial differential, then $\mathcal{Z}^{-}\left(\dot{\beta}^{-}\right)=0$, so $\widetilde{\phi}_{3}$ and $g^{-} d z$ have the same zeros in $\mathbb{C}^{-}$. Since they have the same poles, they are proportionnal, $\widetilde{\phi}_{3}=\lambda g^{-} d z$. Hence the kernel has dimension 1. Since the $\dot{\beta}^{-}$space has dimension $m+1$ and the target space has dimension $m$, the partial differential is onto. The second statement holds because if $\dot{\beta}^{-}=0$, then $\widetilde{\phi}_{3}=-g^{-} d z$ in $\mathbb{C}^{-}$, so they have the same zeros hence $\mathcal{Z}^{-}=0$.

- In the exact same way, the partial differential of $\mathcal{Z}^{+}$with respect to $\dot{\beta}^{+}$is onto and all other partial derivatives of $\mathcal{Z}^{+}$are zero.

- The partial differential of $\left(\mathcal{V}^{A}, \mathcal{V}^{B}\right)$ with respect to $\dot{\gamma}$ is an isomorphism. All other partial derivatives are zero.

Indeed, in term of the new parameters we have $\mathcal{V}_{i}^{A}=-2 \pi \operatorname{Im}\left(\dot{\gamma}_{i}\right)$ and $\mathcal{V}_{i}^{B}=-2 \operatorname{Re}\left(\dot{\gamma}_{i}\right)$, so the partial derivative is injective. Because of the symmetry, the domain and target spaces have the same dimension, namely $m-1$. 
- The partial differential of $\mathcal{H}^{B}$ with respect to $\dot{p}^{+}$is onto. The only other nonzero partial derivatives are the partial differentials with respect to $\dot{\beta}^{+}$ and $\dot{\beta}^{-}$.

Indeed, if $\dot{\beta}^{+}=0$ and $\dot{\beta}^{-}=0$, then $\widetilde{\phi}_{3}=-g^{-} d z$ in $\mathbb{C}^{-}$and $\widetilde{\phi}_{3}=g^{+} d z$ in $\mathbb{C}^{+}$. By equation (9), we get $\mathcal{H}_{i}^{B}=\frac{1}{2}\left(\dot{p}_{m}^{+}-\dot{p}_{i}^{+}\right)$. The statement readily follows.

- The partial differential of $\mathcal{H}^{A}$ with respect to $\left(p_{1}^{-}, \cdots, p_{m-1}^{-}, \gamma_{m}\right)$ is an isomorphism.

To prove this, assume that all parameters but $p^{-}$and $\gamma_{m}$ have their central value. Then $\widetilde{\phi}_{3}=-g^{-} d z$ in $\mathbb{C}^{-}$and $\widetilde{\phi}_{3}=g^{+} d z$ in $\mathbb{C}^{+}$, so formula (8) gives

$$
\begin{aligned}
\mathcal{H}_{i}^{A} & =-\pi \overline{\operatorname{Res}_{p_{i}^{+}}\left(g^{+}\right)^{2}}-\pi \operatorname{Res}_{p_{i}^{-}}\left(g^{-}\right)^{2} \\
& =-2 \pi \sum_{j \neq i} \frac{\gamma_{m}^{2}}{\overline{p_{i}^{+}}-\overline{p_{j}^{+}}}-2 \pi \sum_{j \neq i} \frac{\gamma_{m}^{2}}{p_{i}^{-}-p_{j}^{-}}+2 \pi \frac{\gamma_{m}}{p_{i}^{-}} \\
& =-4 \pi \sum_{j \neq i} \frac{\gamma_{m}^{2}}{\overline{p_{i}^{-}-p_{j}^{-}}}+2 \pi \frac{\gamma_{m}}{p_{i}^{-}} .
\end{aligned}
$$

This implies that

$$
\sum_{i=1}^{m} p_{i}^{-} \mathcal{H}_{i}^{A}=-2 \pi m(m-1) \gamma_{m}^{2}+2 \pi m \gamma_{m}
$$

When $\gamma_{m}$ has its central value, namely $\frac{1}{m-1}$, the right hand side is zero. When $p_{1}^{-}, \cdots, p_{m}^{-}$have their central value, all terms in the left sum are equal by symmetry, so all are zero. This proves that $\mathcal{H}^{A}=0$ at the central value.

Now consider the matrix of the partial differential of $\mathcal{H}^{A}$ with respect to $\left(p_{1}^{-}, \cdots, p_{m-1}^{-}, \gamma_{m}\right)$. Perform the row operation $R_{m} \rightarrow \sum p_{i}^{-} R_{i}$. By the previous formula, we obtain a matrix of the form

$$
\left(\begin{array}{cc}
A & \cdot \\
0 & -2 \pi m
\end{array}\right)
$$

where $A$ is a square matrix of order $m-1$. This matrix is proven to be invertible in appendix A, which proves the statement.

The lemma readily follows from these statements (the matrix of the partial differential has block triangular form).

\subsection{Proof of Theorem 2}

By lemma f 1 and the implicit function theorem, for $\tau$ in a neighborhood of 0 , there exists a smooth function $X(\tau)$ such that $\widetilde{\mathcal{F}}(\tau, X(\tau))=0$. For $t>0$ close 
to zero, let use write $\left(\widetilde{\Sigma}_{t}, \widetilde{g}_{t}, \widetilde{\phi}_{3, t}\right)$ for the Weierstrass data corresponding to the value $\tau=(\log t)^{-1 / 2}$ and $X=X(\tau)$ of the parameters. We choose a base point $z_{0} \in \Sigma$, then this Weierstrass data defines us a minimal immersion $\widetilde{\psi}_{t}$ on $\widetilde{\Sigma}_{t}$ minus the punctures. Let $M_{t}$ be its image. In the following points we prove that the family $\left(M_{t}\right)_{0<t<\varepsilon}$ has all the properties claimed in Theorem 2 .

- $M_{t}$ has $n+1$ catenoidal ends at $q_{1}, \cdots, q_{n-1}, \infty^{-}$and $\infty^{+}$. The logarithmic growths are the opposite of the residue of $\widetilde{\phi}_{3}$ at these points, so by equations (6) and (7), their limit value when $t \rightarrow 0$ are $c_{1}, \cdots, c_{n-1}, 1-$ $\frac{m}{m-1}$ and $\frac{m}{m-1}$. Since we have scaled $M$ so that $c_{n}(M)=1$, this gives formula (2).

- $M_{t}$ converges to $M$ on compact subsets of $\mathbb{R}^{3}$.

This follows from the fact that $\widetilde{g}_{t}$ converges to $g_{0}$ on $\Sigma$ and $\widetilde{\phi}_{3, t}$ converges to $\phi_{3,0}$ on compact subsets of $\Sigma$ minus the punctures, (We translate $M$ so that the image of $z_{0}$ is the origin.)

- $M_{t}$ has non-degenerate Weierstrass Representation.

Indeed, since having maximal rank is an open property, the differential of $\widetilde{\mathcal{F}}$ at $(\tau, X(\tau))$ remains onto for $\tau$ close to 0 . The only issue here is that the parameters $t, p^{ \pm}, \beta^{ \pm}$for $(\widetilde{\Sigma}, \widetilde{g})$ are not the right ones for the definition of non-degeneracy. However, the coordinates that we use on the Hurwitz space are analytic functions of the parameters $t, p^{ \pm}, \beta^{ \pm}$, so if we denote by $\widehat{X}$ the parameters that we use in the definition of non-degeneracy, we have $\widehat{X}=f(\tau, X)$ for some smooth map $f$. Write the equations that we have to solve for non-degeneracy as $\widehat{\mathcal{F}}(\widehat{X})=0$. Then $\widetilde{\mathcal{F}}=\widehat{\mathcal{F}} \circ f$, so the differential of $\widehat{\mathcal{F}}$ is onto.

- $M_{t}$ is embedded.

To prove this statement, we study the asymptotic behavior of $\widetilde{\psi}_{t}$ on each of the domains $\Sigma, \mathbb{C}^{-}$and $\mathbb{C}^{+}$when $t \rightarrow 0$. On $\mathbb{C}^{+}$we have

$$
\lim _{t \rightarrow 0} t \widetilde{\phi}_{1}=-\frac{d z}{2}, \quad \lim _{t \rightarrow 0} t \widetilde{\phi}_{2}=i \frac{d z}{2}, \quad \lim _{t \rightarrow 0} \widetilde{\phi}_{3}=\frac{1}{m-1} \sum_{i=1}^{m} \frac{d z}{z-\omega^{i}} .
$$

Define $\widehat{\psi}_{t}$ on $\mathbb{C}^{+}$as the composition of $\widetilde{\psi}_{t}-\widetilde{\psi}_{t}(0)$ with the affine transformation $\left(x_{1}, x_{2}, x_{3}\right) \mapsto\left(-2 t x_{1},-2 t x_{2}, x_{3}\right)$. Then

$$
\lim _{t \rightarrow 0} \widehat{\psi}_{t}(z)=\left(\operatorname{Re} z, \operatorname{Im} z, u^{+}(z)\right)
$$

where $u^{+}$is the harmonic function

$$
u^{+}(z)=\frac{1}{m-1} \sum_{i=1}^{m} \log \left|z-\omega^{i}\right|
$$

So the image of $\widehat{\psi}_{t}$ converges to the graph of $u^{+}$. For $h$ large enough, the graph of $u^{+}$intersects the plane $x_{3}=-h$ in $m$ closed convex curves, so 
the same is true for the image of $\widehat{\psi}_{t}$ for $t$ small enough. As a conclusion, we can find a height $c_{1}$ (depending on $t$ ) such that the image of $\mathbb{C}^{+}$by $\widetilde{\psi}_{t}$ cuts the plane $x_{3}=c_{1}$ in $m$ closed convex curves. We call $S^{+}$the part which is above this plane. The surface $S^{+}$is embedded (as a graph) and has one upward-going catenoidal end.

In the same way, after horizontal scaling by $-2 t$ and vertical translation, the image of $\mathbb{C}^{-}$by $\widetilde{\psi}$ converges to the graph of $u^{-}(\bar{z})$, where

$$
u^{-}(z)=-\log |z|+\frac{1}{m-1} \sum_{i=1}^{m} \log \left|z-\omega^{i}\right| .
$$

For $h$ large enough, the graph of $u^{-}$intersects the plane $x_{3}=h$ in $m$ closed convex curves and the plane $x_{3}=-h$ in two closed convex curves, one inside the other. Again we may find some heights $c_{2}$ and $c_{3}$, with $c_{3}<c_{2}<c_{1}$ such that for $t$ small enough, the image of $\mathbb{C}^{-}$by $\widetilde{\psi}_{t}$ cuts the plane $x_{3}=c_{2}$ in $m$ closed convex curves and the plane $x_{3}=c_{3}$ in two closed convex curves, one inside the other. Let $S^{-}$be the part bounded by the $m$ top curves and the inside bottom curve. It is an embedded surface with one downward catenoidal end.

Finally, since the top end of $M$ is catenoidal, we may find some height $c_{4}<c_{3}$ such that the image of $\Sigma$ by $\widetilde{\psi}_{t}$ cuts the plane $x_{3}=c_{4}$ in one closed convex curves (and what is above is an annulus). Let $S$ be the part which is below this plane. It is embedded because $M$ is.

The pieces $S, S^{-}$and $S^{+}$are disjoint. (For $S^{-}$and $S$, this uses the maximum principle and the fact that the logarithmic growth of the end of $S^{-}$is larger than the logarithmic growth of the top end of $S$ ). Each component of the complementary set in $M_{t}$ of $S \cup S^{-} \cup S^{+}$is a minimal annulus bounded by two closed convex curves in parallel planes. By a theorem of Shiffman [13], such an annulus is fibered by horizontal curves. It follows that $M_{t}$ is embedded.

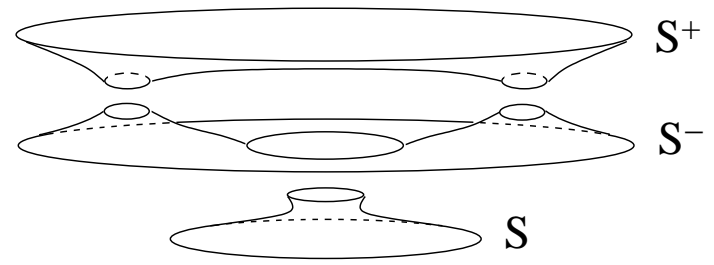

Figure 2: The pieces $S^{+}, S^{-}$and $S$ (in case $M$ is a catenoid).

- For $t$ small enough, the maximum of $|K|$ on $M_{t}$ is greater than $\frac{1}{2}(m-1)^{2}$. Indeed, assume by contradiction that this is not true. Then we can find a sequence $\left(t_{n}\right)_{n}$ converging to zero such that the Gaussian curvature 
on $M_{t_{n}}$ is bounded by $\frac{1}{2}(m-1)^{2}$. Let $A_{n}$ be the image of the annulus bounded by the circles $\left|v_{1}^{+}\right|=\epsilon$ and $\left|v_{i}^{-}\right|=\epsilon$ in $M_{t_{n}}$. Translate $A_{n}$ so that the point where the Gauss map is one is at the origin. The images of the boundary circles are close to circles of radius $O\left(\frac{1}{t_{n}}\right)$, so for any ball $B(0, R)$, $A_{n} \cap B(0, R)$ is properly embedded in $B(0, R)$ for $n$ large enough. As we have uniform Gaussian curvature and area estimate (by the monotonicity formula) for $A_{n}$, a subsequence of $\left(A_{n}\right)_{n}$ converges smoothly on compact subsets of $\mathbb{R}^{3}$ to a complete embedded minimal annulus (12], theorem 4.2.1) hence a catenoid. As the flux of $A_{n}$ converges to $\left(0,0, \frac{2 \pi}{m-1}\right)$, the limit catenoid has waist radius $\frac{1}{m-1}$. Since the maximum of the Gaussian curvature on this catenoid is $(m-1)^{2}$, we have a contradiction.

\section{A A matrix computation}

Lemma 2 Consider an integer $m \geq 2$. Let $\omega=e^{2 \pi i / m}$. Define $p_{i}=\omega^{i}$ for $1 \leq i \leq m$. Consider the order $m-1$ square matrix $A$ defined by

Then $A$ is invertible.

$$
\begin{gathered}
a_{i i}=\frac{m-1}{\left(p_{i}\right)^{2}}-\sum_{\substack{1 \leq j \leq m \\
j \neq i}} \frac{2}{\left(p_{i}-p_{j}\right)^{2}} \\
a_{i j}=\frac{2}{\left(p_{i}-p_{j}\right)^{2}} \quad \text { if } j \neq i
\end{gathered}
$$

Proof. The proof relies on the following observation : if $z \in \mathbb{C}$ is such that $|z|=1$, then

$$
1-2 \operatorname{Re} \frac{1}{(1+z)^{2}}=\frac{2}{|1+z|^{2}}
$$

To see this, observe that the transformation $z \rightarrow \frac{1}{z+1}$ maps the unit circle to the line $\operatorname{Re}(z)=\frac{1}{2}$. If $w$ is on this line, an elementary computation gives $1-2 \operatorname{Re}\left(w^{2}\right)=2|w|^{2}$.

We prove that $A$ has dominant diagonal. We have

$$
\left(p_{i}\right)^{2} a_{i i}=\sum_{j=1}^{m-1}\left(1-\frac{2}{\left(1-\omega^{j}\right)^{2}}\right) .
$$

Since this is a real number,

$$
\begin{gathered}
\left(p_{i}\right)^{2} a_{i i}=\sum_{j=1}^{m-1} \operatorname{Re}\left(1-\frac{2}{\left(1-\omega^{j}\right)^{2}}\right)=\sum_{j=1}^{m-1} \frac{2}{\left|1-\omega^{j}\right|^{2}} \\
\left|a_{i i}\right|=\sum_{j=1}^{m-1} \frac{2}{\left|1-\omega^{j}\right|^{2}}=\sum_{\substack{1 \leq j \leq m-1 \\
j \neq i}}\left|a_{i j}\right|+\frac{2}{\left|p_{i}-1\right|^{2}} .
\end{gathered}
$$

Hence $\left|a_{i i}\right|>\sum_{j \neq i}\left|a_{i j}\right|$ so $A$ is invertible by Hadamard theorem. 


\section{B Deformations of a branched covering}

Let us start with an example to illustrate the problem. We consider the following two deformations of the covering $z \mapsto z^{4}$ of the Riemann sphere :

$$
\begin{gathered}
f_{t}(z)=z^{4}+4 t z^{3}, \\
g_{t}(z)=z^{4}+4 t \alpha z^{3}+4 t^{2} \alpha^{2} z^{2}, \quad \text { with } \alpha^{4}=-27 .
\end{gathered}
$$

By explicit computations, they have the same branching values close to 0 , namely 0,0 and $-3 t$. They are not isomorphic because $f_{t}$ has a branch point of branching order 2 at the origin, whereas $g_{t}$ has three simple branch points (at $0,-t \alpha$ and $-2 t \alpha)$. The conclusion of this example is that in general one cannot parametrize the deformations of a covering with a high order branch point by their branching values.

Definition 4 Fix a value $y_{0}$ in the Riemann sphere. A marked branched covering is a triple $(\Sigma, g, x)$ where $\Sigma$ is a compact Riemann surface, $g$ is a meromorphic function on $\Sigma$ and $x \in \Sigma$ is a regular point of $g$ (i.e. not a branch point) such that $g(x)=y_{0}$.

Two marked branched coverings $(\Sigma, g, x)$ and $\left(\Sigma^{\prime}, g^{\prime}, x^{\prime}\right)$ are isomorphic if there exists a biholomorphic map $\psi: \Sigma \rightarrow \Sigma^{\prime}$ such that $g=g^{\prime} \circ \psi$ and $\psi(x)=x^{\prime}$.

Here we assume for simplicity that there is just one marked point, but there could be several, this does not change anything.

Let $\left(\Sigma_{0}, g_{0}, x_{0}\right)$ be a marked branched covering, $G$ the genus of $\Sigma_{0}$ and $d$ the degree of $g_{0}$. We first construct an explicit family of deformations depending on $2 G+2 d-2$ complex parameters.

Let $p_{1}, \cdots, p_{r}$ be the branch points of $g_{0}$. For $1 \leq i \leq r$, let $k_{i} \geq 2$ be the multiplicity of $g_{0}$ at $p_{i}$ (so the branching order is $k_{i}-1$ ) and $q_{i}=g\left(p_{i}\right)$ the branching value. By using a Moebius transformation, we may assume without loss of generality that all branching values are finite.

We may find $\varepsilon>0$ small enough so that the following holds: for each $1 \leq i \leq r, g_{0}$ is a branched covering of degree $k_{i}$ from a closed topological disk $U_{i}$ containing $p_{i}$ to the closed disk $\bar{D}\left(q_{i}, \varepsilon\right)$. Moreover, the disks $U_{1}, \cdots, U_{r}$ are disjoint, and two disks $\bar{D}\left(q_{i}, \varepsilon\right)$ and $\bar{D}\left(q_{j}, \varepsilon\right)$ are either disjoint or equal. We choose, on the boundary of each disk $U_{i}$, a point $x_{i}$ such that $g_{0}\left(x_{i}\right)=q_{i}+\varepsilon$, which we will use as a marking.

For each $1 \leq i \leq r$, consider the polynomial

$$
h_{i}=z^{k_{i}}+q_{i}+\sum_{j=0}^{k_{i}-2} a_{i j} z^{i} .
$$

If the complex parameters $a_{i j}$ are small enough, all branching values (except $\infty)$ of $h_{i}$ are inside the disk $D\left(q_{i}, \varepsilon\right)$, and $h_{i}$ is a branched covering of degree $k_{i}$ from a closed disk $V_{i}$ to the closed disk $\bar{D}\left(q_{i}, \varepsilon\right)$. We mark this covering with the point $y_{i} \in \partial V_{i}$ such that $h_{i}\left(y_{i}\right)=q_{i}+\varepsilon$ which is closest to $\varepsilon^{1 / k_{i}}$. It depends continuously on the parameters $a_{i j}$ provided they are small enough. 
Then we remove the interior of $U_{i}$ from $\Sigma_{0}$ and replace it by $V_{i}$, indentifying a point $z \in \partial U_{i}$ with the point $z^{\prime} \in \partial V_{i}$ such that $h_{i}\left(z^{\prime}\right)=g_{0}(z)$. Of course, there are $k_{i}$ possible choices for $z^{\prime}$, but the markings allow us to solve this indetermination. More precisely, we may parametrise the boundary of $U_{i}$ as $c(t)$, $t \in\left[0,2 k_{i} \pi\right]$, so that $c(0)=x_{i}$ and $g_{0}(c(t))=q_{i}+\varepsilon e^{i t}$. We may parametrise the boundary of $V_{i}$ as $c^{\prime}(t), t \in\left[0,2 k_{i} \pi\right]$, so that $c^{\prime}(0)=y_{i}$ and $h_{i}\left(c^{\prime}(t)\right)=q_{i}+\varepsilon e^{i t}$. We identify the point $c(t)$ with the point $c^{\prime}(t)$ for all $t$.

Doing this for all $1 \leq i \leq r$ defines a compact Riemann surface which we denote $\Sigma_{a}$, where $a$ denotes the collection of all parameters $a_{i j}, 1 \leq i \leq r$, $0 \leq j \leq k_{i}-2$. Observe that the number of parameters is precisely the total branching order of $g_{0}$, namely $2 G+2 d-2$. On $\Sigma_{a}$ we may define a meromorphic function $g_{a}$ by $g_{a}=g_{0}$ on $\Sigma_{0}$ minus the disks $U_{i}$, and $g_{a}=h_{i}$ on each disk $V_{i}$. Finally, we mark the covering $\left(\Sigma_{a}, g_{a}\right)$ with the point $x_{0}$ we were given (which may be assumed to be outside the disks $U_{i}$ ).

Remark 4 If $k_{i}=2$, then $h_{i}(z)=z^{2}+q_{i}+a_{i 0}$ and the branching value of $g_{a}$ in $V_{i}$ is $q_{i}+a_{i 0}$. So in the case of simple branch points, our parameters are (up to translation by $q_{i}$ ) the branching values, as in the standard parametrisation of the Hurwitz spaces.

Remark 5 The symmetric functions of the branching values of $g_{a}$ inside each $V_{i}$ are polynomial functions of the numbers $a_{i j}$, because the symmetric functions of the branching values of a polynomial may be expressed in function of its coefficients.

Remark 6 The family $\left(\Sigma_{a}\right)_{a}$ is a holomorphic family of compact Riemann surfaces, in the sense that there exists a complex manifold $\mathcal{W}$ of dimension $2 G+2 d-1$ and a holomorphic map $\pi: \mathcal{W} \rightarrow \mathbb{C}^{2 G+2 d-2}$ such that for $a$ close to $0, \pi^{-1}(a)$ is isomorphic to $\Sigma_{a}$.

Remark 7 If $\left(\Sigma_{0}, g_{0}, x_{0}\right)$ is $\sigma$-symmetric, one can define an action of $\sigma$ on $\{1, \cdots, r\}$ by $\sigma\left(p_{i}\right)=p_{\sigma(i)}$. Choose the marking $x_{i}$ so that $x_{\sigma(i)}=\sigma\left(x_{i}\right)$. Then $\left(\Sigma_{a}, g_{a}, x_{0}\right)$ is $\sigma$-symmetric if and only if $a$ satisfies $a_{\sigma(i) j}=\overline{a_{i j}}$ for all $1 \leq i \leq r$ and $0 \leq j \leq k_{i}-2$. Indeed, if $a$ satisfies this condition, then one can define an involution $\widetilde{\sigma}$ on $\Sigma_{a}$ by $\widetilde{\sigma}=\sigma$ on $\Sigma_{0}$ minus the disks $U_{i}$, and for each $1 \leq i \leq r, \widetilde{\sigma}$ maps the point $z \in V_{i}$ to the point $\bar{z}$ in $V_{\sigma(i)}$. It is straightforward to check that $\widetilde{\sigma}$ is well defined and satisfies $g_{a} \circ \widetilde{\sigma}=\overline{g_{a}}$. The "only if" part of the statement is a consequence of uniqueness, proposition 2 below.

We want to prove that with this construction, we obtain all deformations of $\left(\Sigma_{0}, g_{0}\right)$, up to isomorphism of marked coverings. We need the following

Lemma 3 Let $U \subset \mathbb{C}$ be a closed disk and $g: U \rightarrow \bar{D}(0,1)$ be a branched holomorphic covering of degree $k$. The exists a closed disk $V \subset \mathbb{C}$, a biholomorphic mapping $\psi: U \rightarrow V$ and a polynomial $h$ of degree $k$ such that $V=h^{-1}(\bar{D}(0,1))$ and $g=h \circ \psi$. Moreover, $h$ is unique up to composition (on the right) by a transformation of the form $z \mapsto \alpha z+\beta$. 
Proof : choose a point $x_{0}$ in $\partial U$ such that $g\left(x_{0}\right)=1$. We may parametrize the boundary of $U$ as $c(t), t \in[0,2 \pi]$ so that $g(c(t))=e^{k i t}$ and $c(0)=x_{0}$. We glue together $U$ and $\overline{\mathbb{C}} \backslash D(0,1)$ by identifying the point $c(t)$ with the point $e^{i t}$. We obtain a genus zero compact Riemann surface $\widetilde{U}$ on which we can define a meromorphic function $\widetilde{g}$ by $\widetilde{g}=g$ on $U$ and $\widetilde{g}=z^{k}$ on $\overline{\mathbb{C}} \backslash D(0,1)$. By the uniformization theorem, there exists a biholomorphic mapping $\psi: \widetilde{U} \rightarrow \overline{\mathbb{C}}$. We may choose $\psi$ so that $\psi(\infty)=\infty$. Let $h=g \circ \psi^{-1}$. Then $h$ is a meromorphic function on $\overline{\mathbb{C}}$ with a single pole of multiplicity $k$ at infinity, so it is a polynomial of degree $k$. We restrict $\psi$ to $U$ and let $V=\psi(U)$, the first statement of the proposition is proven.

Regarding uniqueness, assume that we have two degree $k$ polynomials $h$ and $h^{\prime}$ such that $D(0,1)$ contains all the branching values of $h$ and $h^{\prime}$ (except infinity) and the branched coverings $h: V \rightarrow \bar{D}(0,1)$ and $h^{\prime}: V^{\prime} \rightarrow \bar{D}(0,1)$ are isomorphic by $\psi: V \rightarrow V^{\prime}$. Then $h: \mathbb{C} \backslash V \rightarrow \mathbb{C} \backslash \bar{D}$ and $h^{\prime}: \mathbb{C} \backslash V^{\prime} \rightarrow \mathbb{C} \backslash \bar{D}$ are isomorphic (unbranched) coverings, so we may extend $\psi$ into $\widetilde{\psi}: \mathbb{C} \rightarrow \mathbb{C}$ such that $h=h^{\prime} \circ \widetilde{\psi}$. Then $\widetilde{\psi}$ is an automorphism of the Riemann sphere which maps $\infty$ to $\infty$, so $\widetilde{\psi}(z)=\alpha z+\beta$.

Next we prove that different choices of the parameters $a$ give non-isomorphic marked coverings.

Proposition 2 If the branched coverings $\left(\Sigma_{a}, g_{a}, x_{0}\right)$ and $\left(\Sigma_{a^{\prime}}, g_{a^{\prime}}, x_{0}\right)$ are isomorphic then $a=a^{\prime}$.

For example, let us consider the branched covering $z \mapsto z^{3}$ and the following two deformations : $z \mapsto z^{3}+t z$ and $z \mapsto z^{3}+t j z$, where $j$ is a primitive cubic root of unity. As branched coverings, they are isomorphic by $z \mapsto j^{2} z$. As marked coverings (with the marking chosen as above) they are not isomorphic.

Proof of the proposition : let $\psi: \Sigma_{a} \mapsto \Sigma_{a^{\prime}}$ be the covering isomorphism. Let $\Omega$ be the Riemann sphere minus the disks $D\left(q_{i}, \varepsilon\right)$. Then by construction, $g_{a}^{-1}(\Omega)=g_{a^{\prime}}^{-1}(\Omega)=g_{0}^{-1}(\Omega)$. So the restriction of $\psi$ to $g_{0}^{-1}(\Omega)$ is an automorphism of the (unbranched) covering $g_{0}: g_{0}^{-1}(\Omega) \rightarrow \Omega$. Since $\psi\left(x_{0}\right)=x_{0}$, it must be the identity. In particular, $\psi\left(x_{i}\right)=x_{i}$ for all $1 \leq i \leq r$, so $\psi$ preserves all the markings.

Then for any $1 \leq i \leq r$, consider the polynomials $h_{i}: V_{i} \rightarrow D\left(q_{i}, \varepsilon\right)$ and $h_{i}^{\prime}: V_{i}^{\prime} \rightarrow D\left(q_{i}, \varepsilon\right)$ which are used to construct respectively $\Sigma_{a}$ and $\Sigma_{a^{\prime}}$. Then $h_{i}=h_{i}^{\prime} \circ \psi$ on $V_{i}$, so by the uniqueness part of the lemma, the restriction of $\psi$ to $V_{i}$ has the form $z \mapsto \alpha z+\beta$. As $h_{i}$ and $h_{i}^{\prime}$ are unitary polynomials, we must have $\alpha^{k_{i}}=1$. Since they have no term of degree $k_{i}-1$, we must have $\beta=0$. Since $\psi$ preserves the markings, $\alpha=1$ by our choice of the markings of the polynomials. Hence $h_{i}=h_{i}^{\prime}$ for all $1 \leq i \leq r$, which means that $a=a^{\prime}$.

By a deformation of $\left(\Sigma_{0}, g_{0}\right)$, we mean the following. Let $S$ be $\Sigma_{0}$ seen as a differentiable 2-manifold, i.e. forgetting the conformal structure. Let $\left(g_{t}\right)_{0 \leq t \leq 1}$ be a continuous family of branched coverings from $S$ to $\overline{\mathbb{C}}$. It is well known that each $g_{t}$ induces a conformal structure on $S$ which we denote by $\Sigma_{t}$. We also assume that each $g_{t}$ is close enough to $g_{0}$ in the sense that all the branching values of $g_{t}$ remain inside the union of the disks $D\left(q_{i}, \varepsilon\right)$. Then we say that each 
$\left(\Sigma_{t}, g_{t}\right)$ is a deformation of $\left(\Sigma_{0}, g_{0}\right)$. If $\left(\Sigma_{0}, g_{0}\right)$ is marked with the point $x_{0}$, then each $\left(\Sigma_{t}, g_{t}\right)$ admits a unique marking $x_{0}(t)$, depending continuously on $t$, such that $g_{t}\left(x_{0}(t)\right)=g_{0}\left(x_{0}\right)$.

Proposition 3 If $(\Sigma, g)$ is a deformation of $\left(\Sigma_{0}, g_{0}\right)$, there exists $a \in \mathbb{C}^{2 G+2 d-2}$ such that $(\Sigma, g)$ is isomorphic to $\left(\Sigma_{a}, g_{a}\right)$.

Proof : let $\Omega$ be the complement in the Riemann sphere of the disks $D\left(q_{i}, \varepsilon\right)$. Then the restrictions $g_{0}: g_{0}^{-1}(\Omega) \rightarrow \Omega$ and $g: g^{-1}(\Omega) \rightarrow \Omega$ are isomorphic (unbranched) coverings so there exists an isomorphism $\psi: g_{0}^{-1}(\Omega) \rightarrow g^{-1}(\Omega)$ such that $g_{0}=g \circ \psi$. We can easily extend $\psi$ to those components of $\Sigma_{0} \backslash g_{0}^{-1}(\Omega)$ in which $g_{0}$ has no branch point. Indeed, if $U$ is such a component, let us call $U^{\prime}$ the component of $\Sigma \backslash g^{-1}(\Omega)$ which is bounded by $\psi(\partial U)$. Then $g_{0}$ and $g$ are diffeomorphisms from respectively $U$ and $U^{\prime}$ to the same disk $D\left(q_{i}, \varepsilon\right)$, so we may extend $\psi$ as $\left(\left.g\right|_{U^{\prime}}\right)^{-1} \circ g_{0}$ on $U$.

Finally, let us consider one of the disks $U_{i}$ in which $g_{0}$ has a branch point of mutiplicity $k_{i}$. Let $U_{i}^{\prime}$ be the component of $\Sigma \backslash g^{-1}(\Omega)$ which is bounded by $\psi\left(\partial U_{i}\right)$. Then $U_{i}^{\prime}$ is a disk and $g: U_{i}^{\prime} \rightarrow D\left(q_{i}, \varepsilon\right)$ is a branched covering of degree $k_{i}$ (altough the branch point may have split into several ones). Recall that we have a marking $x_{i} \in \partial U_{i}$, and let us call $x_{i}^{\prime}=\psi\left(x_{i}\right) \in \partial U_{i}^{\prime}$. By the lemma, there exists a polynomial $h_{i}$ of degree $k_{i}$ and a diffeomorphism $\psi_{i}: U_{i}^{\prime} \rightarrow V_{i}$ such that $g=h_{i} \circ \psi_{i}$ on $U_{i}^{\prime}$. By composing $h_{i}$ on the right by a transformation $z \mapsto \alpha z+\beta$, we may assume that $h_{i}$ is a unitary polynomial with no term of degree $k_{i}-1$, and moreover, $\psi_{i}\left(x_{i}^{\prime}\right)$ is the solution of $h_{i}(z)=q_{i}+\varepsilon$ which is closest to $\varepsilon^{1 / k_{i}}$. Let $\left(\Sigma_{a}, h_{a}\right)$ be the branched covering constructed with the polynomials $h_{1}, \cdots, h_{r}$ we have found. Define $\widetilde{\psi}: \Sigma_{a} \rightarrow \sum$ by $\widetilde{\psi}=\psi$ on $\Sigma_{0}$ minus the disks $U_{1}, \cdots, U_{r}$ and $\widetilde{\psi}=\psi_{i}^{-1}$ on each $V_{i}$. Then $\widetilde{\psi}$ is an isomorphism between the marked coverings $\left(\Sigma_{a}, g_{a}\right)$ and $(\Sigma, g)$.

\section{References}

[1] P. Collin, R. Kusner, W. H. Meeks III, and H. Rosenberg. The geometry, conformal structure and topology of minimal surfaces with infinite topology. J. Differential Geom., 67:377-393, 2004. MR2153082, Zbl 1098.53006.

[2] John D. Fay. Theta functions on riemann surfaces. Springer Verlag, Lecture notes in mathematics, No 352, 1973. MR0335789, Zbl 0281.30013.

[3] W. Fulton. Hurwitz schemes and irreducibility of moduli of algebraic curves. Ann. of Math., 90(2):542-575, 1969. MR0260752, Zbl 194.21901.

[4] L. Hauswirth and F. Pacard. Higher genus Riemann minimal surfaces. Inventiones Mathematicae, 169(3):569-620, 2007. MR2336041, Zbl 1129.53009 .

[5] D. Hoffman, H. Karcher, and F. Wei. The genus one helicoid and the minimal surfaces that led to its discovery. In Global Analysis and Modern 
Mathematics. Publish or Perish Press, 1993. K. Uhlenbeck, editor, p. 119170. MR1278754, Zbl 990.54130.

[6] H. Masur. The extension of the weil petersson metric to the boundary of teichmuller space. Duke Math. J., 43:623-635, 1976. Zbl 0358.32017.

[7] L. Mazet, M.M. Rodríguez, and M. Traizet. Saddle towers with infinitely many ends. Indiana Univ. Math. J., 56(6):2821-2838, 2007. MR2375703.

[8] L. Mazet and M. Traizet. A quasi-periodic minimal surface. Commentarii mat. Helv., 83:573-601, 2008.

[9] W. H. Meeks III, J. Pérez, and A. Ros. The geometry of minimal surfaces of finite genus II; nonexistence of one limit end examples. Invent. Math., 158:323-341, 2004. MR2096796, Zbl 1070.53003.

[10] N. Nadirashvili. Hadamard's and Calabi-Yau's conjectures on negatively curved and minimal surfaces. Invent. Math., 126(3):457-465, 1996. MR1419004, Zbl 0881.53053.

[11] J. Pérez and A. Ros. The space of properly embedded minimal surfaces with finite total curvature. Indiana Univ. Math. J., 45(1):177-204, 1996. MR1406689, Zbl 864.53008.

[12] J. Pérez and A. Ros. Properly embedded minimal surfaces with finite total curvature. In The Global Theory of Minimal Surfaces in Flat Spaces, pages 15-66. Lecture Notes in Math 1775, Springer-Verlag, 2002. G. P. Pirola, editor. MR1901613, Zbl 1028.53005.

[13] M. Shiffman. On surfaces of stationary area bounded by two circles, or convex curves, in parallel planes. Ann. of Math., 63:77-90, 1956. MR0074695, Zbl 0070.16803 .

[14] M. Traizet. An embedded minimal surface with no symmetries. J. Differential Geometry, 60(1):103-153, 2002. MR1924593, Zbl 1054.53014. 